\title{
Gradual spontaneous resolution of corneal bloodstaining after anterior chamber irrigation for rebleeding secondary to traumatic hyphema
}

This article was published in the following Dove Press journal: International Medical Case Reports Journal

\author{
Hsin-Ying Lin \\ Kai-Ling Peng \\ Department of Ophthalmology, \\ Chi Mei Medical Center, Tainan, Taiwan
}

Correspondence: Kai-Ling Peng Department of Ophthalmology, Chi Mei Medical Center, No. 901, Zhonghua Road, Yongkang District, Tainan 7I0, Taiwan

Tel +886 $62812811 \times 57247$

Email caropk165@gmail.com

\begin{abstract}
Corneal bloodstaining, which is brown or dark yellow in color, is induced by hemoglobin deposition, and its breakdown products extend into the corneal stroma. In this article, we report a rare case of corneal bloodstaining induced by total hyphema after rebleeding for traumatic hyphema. The patient underwent irrigation of the anterior chamber (AC) and cataract surgery of the right eye after trauma. After oral and topical treatment the imprint of corneal bloodstaining faded, and it nearly disappeared after the procedures. Corneal bloodstaining is undoubtedly a vision-threatening complication of total hyphema after ocular trauma, surgical intervention, and even rebleeding. Removal of the total hyphema as soon as possible decreases the severity of corneal bloodstaining, shortens the course of spontaneous healing, and thus improves vision. Keywords: corneal bloodstaining, total hyphema, traumatic hyphema, rebleeding, corneal imprint
\end{abstract}

\section{Introduction}

The incidence of corneal bloodstaining created by hemoglobin deposition and its breakdown products that extend into the corneal stroma has been reported at $2 \sim 11 \%$ after traumatic hyphema ${ }^{1-4}$ and the accumulation of blood in the aqueous fluid of the anterior chamber (AC) by injury to the tissue of iris or ciliary body. The process of corneal bloodstaining has been described that hemoglobulin particles in the hyphema concentrated and diffused into the corneal stroma even across an intact Descemet's membrane. ${ }^{5}$

We report herein a rare case of corneal bloodstaining secondary to rebleeding after traumatic hyphema in a male patient. His blood-stained cornea spontaneously and totally resolved gradually after the removal of rebleeding hyphema and cataract surgery with obvious vision improvement.

\section{Case report}

A 56-year-old healthy male without systemic diseases was suddenly hit by a stone in his right eye while working. A day later, he presented at our branch hospital where his best-corrected visual acuity (BCVA) was 3/20 in his right eye and 12/20 in his left eye. The IOP was $16 \mathrm{mmHg}$ in his right eye and $16 \mathrm{mmHg}$ in his left eye. Under a slit lamp, the right cornea showed an abrasion of $3 \mathrm{~mm} \times 3 \mathrm{~mm}$ in size with positive fluorescein staining. Hyphema with red blood cells ++ floating in the right AC was at a level of 1/2 height. Simultaneous temporal iris paralysis with a droplet-shaped pupil was noted. He was treated with topical steroids, antibiotics, and bed rest in a semi-Fowler position. 
Six days after trauma, the patient was presented at our emergency room with a total loss of vision in his right eye. His BCVA was only light sense in his right eye. The IOP was $20 \mathrm{mmHg}$ in his right eye and $6 \mathrm{mmHg}$ in his left eye. A slit lamp showed total hyphema with dark red color extending into the corneal stroma of the right eye (Figure 1). A B-scan showed that the retina was attached without vitreous opacity. He was administered hemostatic oral medications. However, total hyphema in his right eye lasted for a week.

Fourteen days after the trauma, the patient underwent irrigation of the $\mathrm{AC}$ of his right eye. Severe corneal stromal edema with striae and central corneal bloodstaining were noted on the first postoperative day (Figure 2A). Oral prednisolone $(10 \mathrm{mg} /$ day), topical steroids ( $1 \%$ prednisolone acetate ophthalmic suspension qid), topical antibiotics (4\% sulfamethoxazole qid), and mydriatics ( $1 \%$ atropine sulfate bid) were prescribed during the first 2 weeks after surgery. Oral (prednisolone $5 \mathrm{mg} /$ day) and topical steroids $(0.3 \%$ betamethasone sodium phosphate qid) were further tapered following the gradual resolution of the corneal edema and residual opaque blood clot at the temporal half of the $\mathrm{AC}$ (Figure 2B). The corneal bloodstaining imprint remained 1 month after surgery, while the corneal edema was completely resolved (Figure 2C).

Maintaining topical steroids $(0.3 \%$ betamethasone sodium phosphate tid) with additional lubricants and the range and color of the corneal bloodstaining became smaller, thinner, and shallower 6 weeks later (Figure 2D). The BCVA of the right eye improved to 4/20 3 months thereafter.

Although the corneal bloodstaining faded, the anterior subcapsular opacity of the lens in the patient's right eye became so severe (Figure 2E) that he underwent right eye cataract surgery 6 months after AC irrigation. Ten months later, the BCVA of his right eye improved to $8 / 20$ with centrally disappearing corneal bloodstaining (Figure 3).
Fifteen months after AC irrigation, the BCVA of his right eye eventually reached $14 / 20$ with correction.

\section{Discussion}

Several studies elucidated that a prolonged high IOP, a persistent and large amount of hyphema, or corneal endothelial dysfunction may increase the risk of corneal bloodstaining. $2,4,5$ In our case, total hyphema without high IOP still induced corneal bloodstaining, which occurred after AC irrigation of the total hyphema for rebleeding. Yang et $\mathrm{al}^{6}$ also reported the spontaneous resolution of corneal bloodstaining without increased IOP secondary to total hyphema after trabeculectomy. We speculated that high IOP might be a contributing factor instead of an essential factor for corneal bloodstaining. However, total hyphema seemed to be the critical factor, with an incidence of $33-100 \%{ }^{4}$

During AC irrigation, we found that most of the hyphema was liquid but stickier and darker than fresh hyphema. It is reasonable that the concentration of hemoglobin and breakdown products would persistently increase after total hyphema because the aqueous part may constantly drain while the site continues to bleed. The concentrated hemoglobin and breakdown products in the $\mathrm{AC}$ accelerate their diffusion directly and passively to the posterior corneal stroma. However, the solid status of the total hyphema at the side of the endothelium may not induce corneal bloodstaining since coagulated blood clots do not move. Compared to the dense color of the corneal bloodstaining in patients of Yang et $\mathrm{al}^{6}$ whose total hyphema was removed 1 month later, and Fraser et al, ${ }^{7}$ whose total hyphema spontaneously resolved 1 month later, our corneal bloodstaining was obviously lighter since the total hyphema lasted for only 2 weeks. Removing the total liquid hyphema as soon as possible might decrease the concentration of hemoglobin and breakdown products, thus lessening the severity and coloration of the corneal bloodstaining.

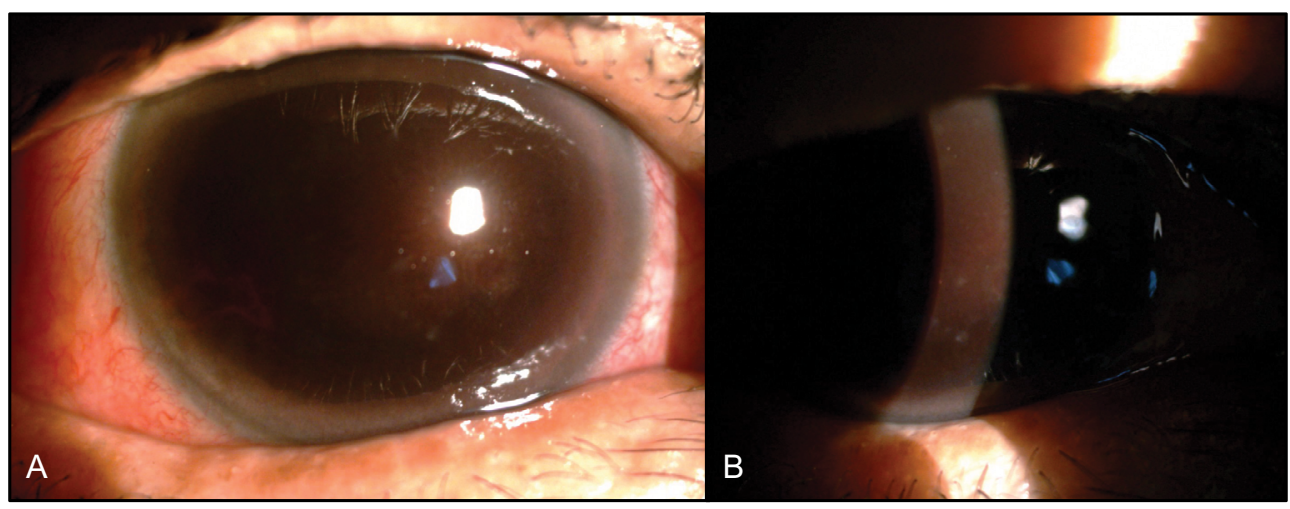

Figure I A slit lamp image showed the total hyphema with a dark red color expanded into the cornea stroma of the right eye from anterior view (A) and oblique view (B). 


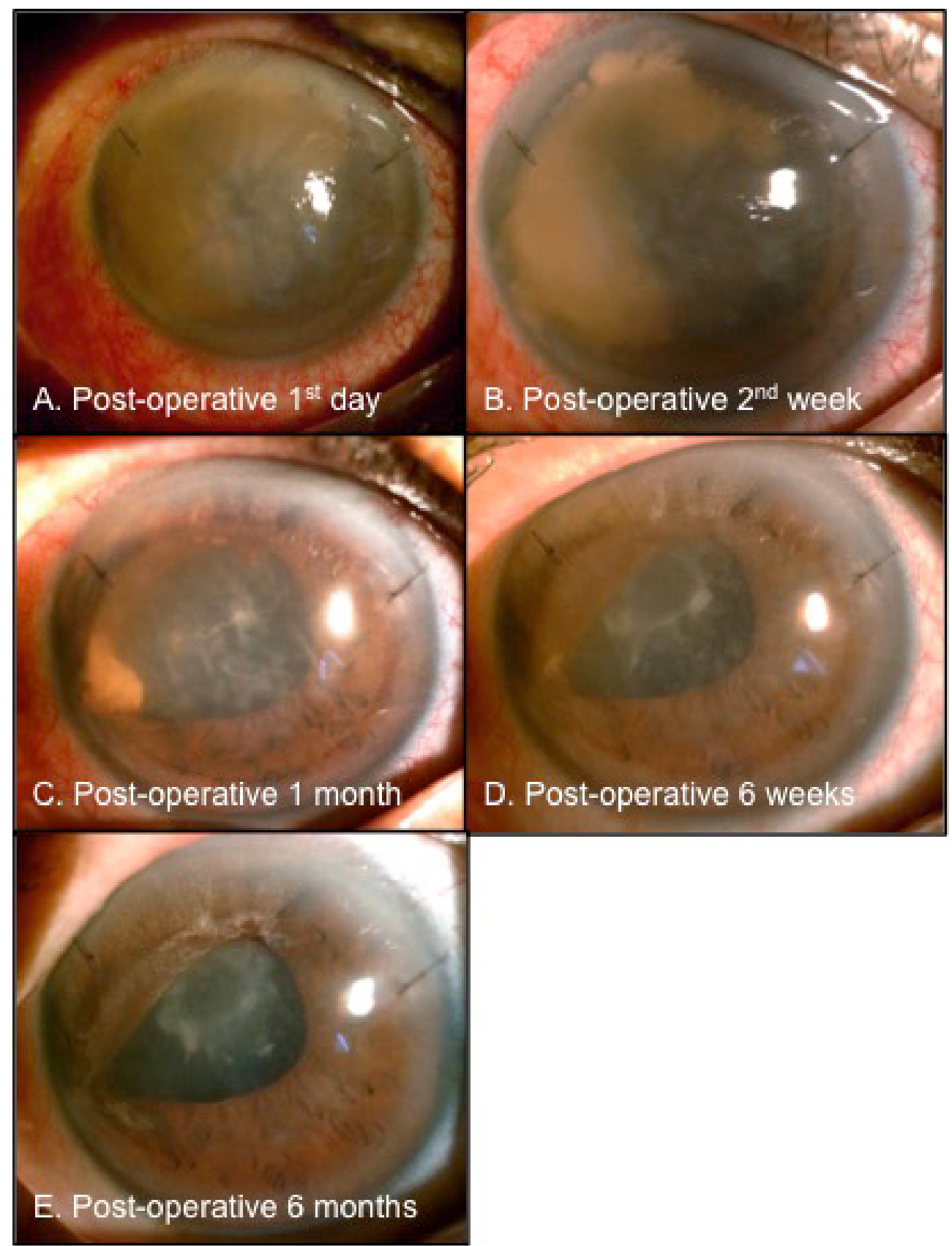

Figure 2 After removal of the total hyphema, the corneal bloodstaining cleared centrifugally from the peripheral to the central corneal stroma and from the posterior to the anterior corneal stroma.

Notes: (A) postoperative first day; (B) postoperative second week; (C) postoperative I month; (D) postoperative 6 weeks; and (E) postoperative 6 months.

The mechanism of spontaneous resolution of corneal bloodstaining involves macrophages from the limbal blood vessels first aggregating from the peripheral cornea and then slowly migrating into the central cornea to clear the erythrocyte debris. ${ }^{6}$ In our patient, corneal bloodstaining spontaneously cleared centrifugally from the peripheral to the central area and from the back to the front gradually during
15 months after rebleeding. Instead of waiting for the longterm spontaneous resolution of corneal bloodstaining, Yang et $a l^{6}$ demonstrated that a conjunctival flap with transplanted vessels covering the central corneal bloodstaining played a significant role in shortening the interval of clearing up for delivering macrophages directly to the central cornea. Even after placing a conjunctival flap with transplanted vessels, 


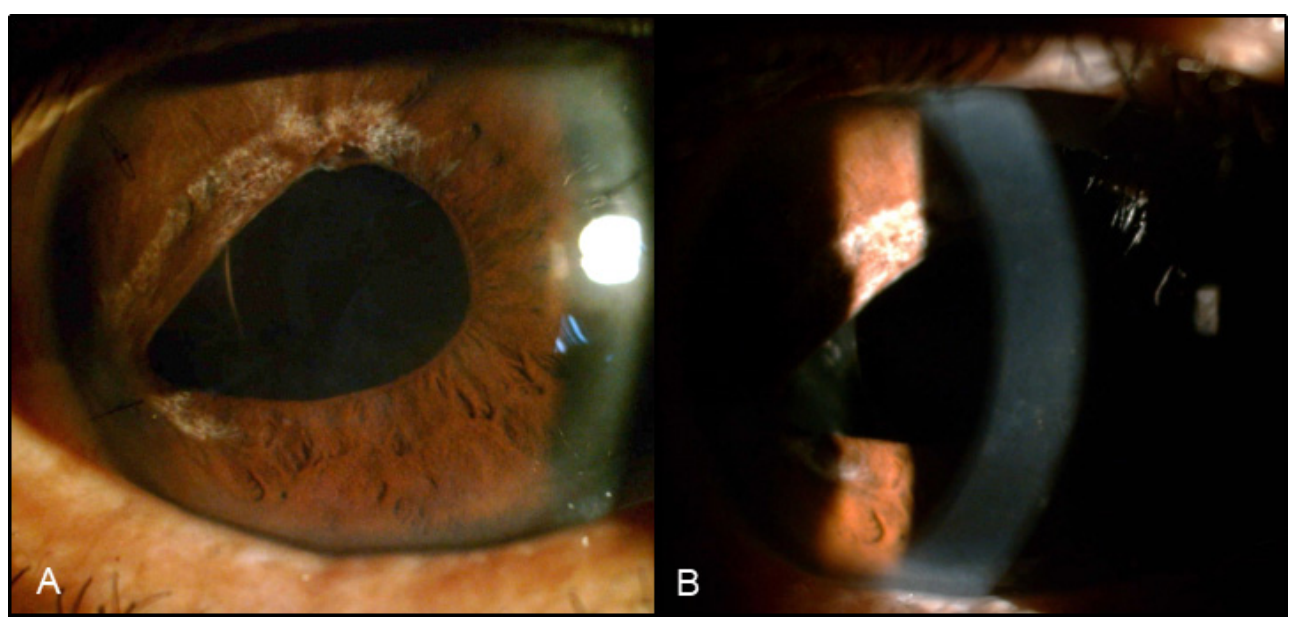

Figure 3 After cataract surgery, the corneal bloodstaining seemed to nearly disappear in the center 10 months after AC irrigation with sparse anterior stromal traces from anterior view $(A)$ and oblique view (B).

Abbreviation: AC, anterior chamber.

it took 14 months for their patients to completely resolve, nearly the same recovery time as our patient, although the coloration of the corneal bloodstaining in their case was denser. A conjunctival flap with vessels might be a better modality for accelerating the clearance of corneal bloodstaining in severe cases.

In conclusion, corneal bloodstaining is undoubtedly a vision-threatening complication of total hyphema after ocular trauma, surgical intervention, and even rebleeding. Control of IOP is important to prevent optic nerve damage. Furthermore, removing the whole hyphema as soon as possible will decrease the severity of corneal bloodstaining, shorten the course of clearing up spontaneously, and thus improve vision.

\section{Consent for publication}

Written informed consent was obtained from the patient for publication of this case report and the accompanying images.

\section{Data sharing statement}

All the data supporting our findings will be shared upon request, although the majority is contained within the manuscript.

\section{Disclosure}

The authors report no conflicts of interest in this work.

\section{References}

1. Mcdonnell PJ, Gritz DC, Mcdonnell JM, Zarbin MA. Fluorescence of blood-stained cornea. Cornea. 1991;10(5):445-449.

2. Brodrick JD. Corneal blood staining after hyphaema. Br J Ophthalmol. 1972;56(8):589-593.

3. Bansal S, Gunasekeran DV, Ang B, et al. Controversies in the pathophysiology and management of hyphema. Surv Ophthalmol. 2016;61(3):297-308.

4. Walton W, von Hagen S, Grigorian R, Zarbin M. Management of traumatic hyphema. Surv Ophthalmol. 2002;47(4):297-334.

5. Messmer EP, Gottsch J, Font RL. Blood staining of the cornea: a histopathologic analysis of 16 cases. Cornea. 1984;3(3):205-212.

6. Yang X, Zhou Q, du S. Conjunctival flap covering in the treatment of corneal blood staining. Can J Ophthalmol. 2011;46(5):442-443.

7. Fraser C, Liew S, Fitzsimmons R, Arnold J. Spontaneous resolution of corneal blood staining. Clin Exp Ophthalmol. 2006;34(3):279-280.

\section{Publish your work in this journal}

The International Medical Case Reports Journal is an international, peer-reviewed open-access journal publishing original case reports from all medical specialties. Previously unpublished medical posters are also accepted relating to any area of clinical or preclinical science. Submissions should not normally exceed 2,000 words or

\section{Dovepress}

4 published pages including figures, diagrams and references. The manuscript management system is completely online and includes a very quick and fair peer-review system, which is all easy to use. Visit $\mathrm{http}: / / \mathrm{ww}$.dovepress.com/testimonials.php to read real quotes from published authors. 